\title{
Polydrug Use and Heterogeneity in HIV Risk Among People Who Inject Drugs in Estonia and Russia: A Latent Class Analysis
}

\author{
Isabel Tavitian-Exley ${ }^{1} \cdot$ Marie-Claude Boily $^{1} \cdot$ Robert Heimer $^{2} \cdot$ Anneli Uusküla $^{3}$ • \\ Olga Levina $^{4} \cdot$ Mathieu Maheu-Giroux 5
}

Published online: 11 July 2017

(C) The Author(s) 2017. This article is an open access publication

\begin{abstract}
Non-medical drug injection is a major risk factor for HIV infection in Russia and Estonia. Multiple drug use (polydrug) has further been associated with increased harms. We compared HIV, injecting and sexual risk associated with polydrug use among people who injected drugs (PWID) in 2012-2013 in Kohtla-Järve (Estonia, $\mathrm{n}=591$ ) and St Petersburg (Russia, $\mathrm{n}=811$ ). Using latent class analysis, we identified five (poly)drug classes, the largest consisting of single-drug injectors among whom an opioid was the sole drug injected (56\% of PWID). The four remaining polydrug classes included polydrug-polyroute injectors who injected and used opiates and stimulants (9\%), opiate-stimulant poly-injectors who injected amphetamine-type-stimulants with a primary opiate $(7 \%)$ and opiate-opioid poly-injectors who injected opioids and opiates (16\%). Non-injection stimulant co-users were injectors who also used non-injection stimulants (12\%). In multivariable multinomial regressions, all four polydrug
\end{abstract}

Electronic supplementary material The online version of this article (doi:10.1007/s10461-017-1836-0) contains supplementary material, which is available to authorized users.

Isabel Tavitian-Exley

Tavitianexley@gmail.com

$\triangle$ Marie-Claude Boily

mc.boily@imperial.ac.uk

1 Department of Infectious Disease Epidemiology, Imperial College London, Norfolk place, London W21PG, UK

2 Epidemiology of Microbial Diseases, School of Public Health, Yale University, New Haven, USA

3 Faculty of Medicine, University of Tartu, Tartu, Estonia

4 NGO Stellit, St Petersburg, Russian Federation

5 Department of Epidemiology, Biostatistics and Occupational Health, McGill University, Montréal, Canada classes were associated with greater injection risks than single-drug injection, while opiate-stimulant and opiateopioid poly-injection were also associated with having multiple sex partners. Riskier behaviours among polydruginjectors suggest increased potential for transmission of blood-borne and sexually-transmitted infections. In addition to needles/syringes provision, services tailored to PWID drug and risk profiles, could consider drug-appropriate treatment and sexual risk reduction strategies to curb HIV transmission.

Resumen La inyección de drogas no médicas es un factor principal de riesgo para la infección por el VIH en Rusia y Estonia. Además, el uso de múltiple drogas (poli-drogas) se ha asociado con un aumento de daños. Comparamos el estado de infección de VIH, y los comportamientos del riesgo de inyección y sexual asociado con el uso de poli-drogas entre las personas que inyectan drogas (PID) en 2012-2013 en Kohtla-Järve (Estonia, $\mathrm{n}=591$ ) y San Petersburgo (Rusia, $\mathrm{n}=811$ ). Utilizando el análisis de la clase latente, identificamos cinco clases de uso de (poli)drogas, el más grande constituido por inyectores de una solo droga, inyectando un único opioide (56\%). Las cuatro clases restantes incluyeron inyectores de poli-drogas/poli-rutas que inyectaron y usaron opiáceos y estimulantes (9\%), poli-inyectores de opiáceo-estimulante que inyectaron estimulantes de tipo anfetamina con un opiáceo primario (7\%) y poli-inyectores de opiáceoopioides que inyectaron opiáceos y opioides (16\%). Los cousuarios de estimulantes no inyectables eran inyectores que también usaban estimulantes no inyectables (12\%). En las regresiones multinomiales multivariables, las cuatro clases de uso de poli-droga se asociaron con mayores riesgos de inyección que la inyección de un solo fármaco, mientras que la poli-inyección de opiáceo-estimulante y la poli-inyección de opiáceos-opioides también se asociaron con tener 
múltiples parejas sexuales. Comportamientos más riesgosos entre los inyectores de poli-drogas sugieren un mayor potencial para la transmisión de infecciones transmitidas sexual y por la sangre. Además de la provisión de agujas y jeringas, los servicios adaptados a los perfiles de riesgo en las PID podrían considerar incluir estrategias de tratamiento y de reducción de riesgo de transmisión sexual adecuadas para los usadores de diferentes drogas, para reducir la transmisión del VIH.

Keywords HIV - Risk behaviours - People who inject drugs · Polydrug use · Heroin/opioid · Amphetamines . Eastern Europe $\cdot$ Latent class analysis

\section{Introduction}

Non-medical drug injection has been a driver of HIV and hepatitis C (HCV) epidemics in Eastern Europe and central Asia, where people who inject drugs (PWID) were estimated to account for half of new HIV and a majority of HCV infections in 2014 [1-5]. Estonia and Russia in particular have reported some of the highest HIV prevalence in key populations-populations disproportionately affected by HIV-outside of sub-Saharan Africa [1, 6-11]. HIV seropositivity among PWID was 53\% in Tallinn, the capital of Estonia, and $70 \%$ in Kohtla-Järve, the fifth largest city in the country in 2007 [12-14]. In the Russian Federation, HIV prevalence ranged from $9 \%$ to $64 \%$ among PWID and was 59\% among PWID in St Petersburg in 2009 [15, 16].

Kohtla-Järve and St Petersburg have epidemiologically comparable epidemics of drug use and HIV. Both cities are situated on the northern part of two major heroin trafficking corridors linking Afghanistan to the heroin markets of Western Europe [3, 17]. Although a majority of PWID in the region inject heroin or synthetic opioids (e.g. fentanyl, methadone) [16, 18, 19], Estonia reported one of the highest prevalence of amphetamine-type stimulant (ATS) use in Europe [15, 20]. Data further suggest that ATS has become a major secondary drug among PWID in KohtlaJärve and St Petersburg [18, 21-23]. Polydrug use consists of the concurrent use of multiple illicit drugs and such practices have recently been facilitated by increased drug availability. These trends are worrisome as polydrug use among PWID has been associated with a greater risk of injury, infection, overdose and drug-induced deaths in many parts of the world [10, 24-26]. Stimulants have been associated with greater injecting and sexual risks, and are known to adversely affect drug treatment outcomes and adherence to antiretroviral therapy among PWID [24, 27-29]. Combining stimulants with opioids, for example, may lead to riskier behaviours and HIV infection among PWID, with potentially important implications for prevention and treatment programmes [29-31]. However, evidence on polydrug use and HIV risk remains limited and no epidemiological studies have examined the extent to which heterogeneity in drug injection among PWID in Eastern Europe is related to risk behaviours and prevalence of HIV infection [32-36].

Uncovering patterns of polydrug use can be challenging. Latent class analysis (LCA) has been used to empirically identify classes of individuals, based on a set of observed characteristics [37, 38]. So far, most LCA studies have been conducted among PWID who injected heroin and cocaine in the United States and Canada, and few of them have examined the relationship between polydrug use and HIV risk behaviours outside the North American context [39-41]. In our study, polydrug use was defined as injecting a main illicit drug and injecting or using one or more additional illicit substance (excluding cannabis or alcohol). Our study objectives were (i) to empirically identify classes of polydrug use on the basis of drug class and administration route, and (ii) to investigate whether injecting and sexual risk behaviours, demographic factors, HIV and HCV are associated with different categories of (poly)drug use among PWID.

\section{Methods}

\section{Study Population}

PWID were recruited between May and July 2012 in Kohtla-Järve and from November 2012 to June 2013 in St Petersburg, using respondent driven sampling (RDS), a variant of chain referral sampling [42-44]. Comparable recruitment criteria (men and women aged 18 or over, "having injected drugs in the past 30 days", lived in St Petersburg or Kohtla-Järve and had provided informed consent for the study), survey methodology and questionnaires were used, details of which have been previously published [10, 16, 23, 45-47]. In brief, six seeds representing diverse PWID sub-groups in terms of gender, main drug used, age and HIV status, were selected through outreach programmes in Kohtla-Järve, and 16 seeds in St Petersburg. Each seed recruited up to three PWID from their personal network, who on completing the survey recruited a maximum of three new participants [10, 44, 48]. Recruitment was tapered once the HIV outcome converged to a sample equilibrium and the target sample size was reached [43]. The samples were recruited over 11 waves in Kohtla-Järve and 12 waves in St Petersburg.

\section{Measures}

The study questionnaire for both sites included standardised items from established tools including the WHO Drug Injecting study Phase II survey (v2b) for risk behaviours 
$[10,49]$. Information on social and demographics, injection and sexual risk behaviours, testing and access to harm reduction services was elicited in a structured confidential face-to-face interview using a questionnaire administered by trained fieldworkers.

The variables examined for association with polydrug classes included past month injecting risk, past 6 months sexual risk behaviour and serological markers for HIV, HCV and Herpes Simplex Virus-2 (HSV-2) infection. Key behavioural variables predictive of HIV and viral hepatitis transmission were examined: injecting frequency ( $\geq$ daily vs. $<$ daily), injecting intensity ( $\geq 2$ day vs. $<2$ day on last day injected), sharing needles and syringes, sharing drug paraphernalia, back-loading (filling a syringe from another working syringe), multiple sex partners, having a sex partner who injected drugs and having been paid for sex [50]. The variable "any sex in the last 6 months" was used to exclude non-sexually active PWID when examining associations. Demographic and contextual variables included age, sex, ethnicity (non-Russian/ethnic Russian), living arrangements (unstable/stable), source of income (nonregular/salaried), contact with needle and syringe programme (NSP), past year drug substitution treatment (OST) and city (Kohtla-Järve/St Petersburg). HIV serostatus was assessed with HIV antigen/antibody combo-assay (ADVIA-Centaur, Siemens healthcare diagnostics) and HIV-I/II Score line immunoassay confirmatory test (INNOLIA $^{\circledR}$, Fujirebio Europe) in Kohtla-Järve. Rapid oral HIVI/II antibody tests were used in St Petersburg (OraQuick Advance $^{\circledR}$, OraSure Technologies Inc.) and confirmed at the City AIDS Centre [10]. HCV and HSV reactivity were measured in Kohtla-Järve only, using commercially available anti-HCV (Murex v4.0) and HSV-2 IgG ELISA kits (IBL International $\mathrm{GmbH}$ ).

\section{Statistical Analyses}

LCA was used to identify PWID subgroups with similar patterns in primary (main), and additional drug(s) injected or used (i.e. polydrug classes) [51]. LCA is a form of latent variable modelling which aims to identify underlying relationships in a defined set of observed variables to divide a heterogeneous population into more homogenous subgroups (latent classes), by grouping observations that display similar response patterns on these variables $[37,38]$. LCA methodology is particularly useful and was selected above other methods such as cluster analysis or factor analysis for its ability to generate model-based class characterisations with conditional probabilities.

Seven variables of interest describing characteristics of polydrug use in the past month were used in our LCA. These included the main drug class injected (ATS or opiate/opioid), injection of additional opiate/opioid, injection of additional stimulant, use of additional opiate/opioid, use of additional stimulant, number of drugs injected and number of non-injection drugs used (Supplementary material, Figure S1). The seven drug use variables were entered into a latent class model and fitted to the data, starting with one class and progressively increasing the number of classes to six.

The selection of the best model was informed by several fit statistics, current epidemiology of drug use, meaningfulness and practical implications of classes. The fit statistics considered were Pearson's Chi squared and Log likelihood ratio tests (LR), Lo-Mendell-Rubin likelihood ratio test for nested models (LRMT) and the Akaike (AIC) and Bayesian information criteria (BIC) [38, 52, 53]. The two RDS samples were jointly analysed, based on similarities in primary drugs injected, frequency of polydrug use and our objective of identifying common drug combinations across settings (Table S1). A dummy variable for city was included in the model as a covariate. That is, city effects were adjusted for in the LCA, but city was not included alongside the seven variables forming the latent classes. The LCA assumption of conditional independence was ascertained by examining bivariate residuals for each set of variables in LCA [54-56]. LCA was performed using Mplus version 7.4 [55].

Socio-demographic, programme and HIV risk behaviour variables were then compared between emergent sub-types of the best fit latent class model, in univariate and multivariate multinomial logistic regression [57, 58]. Pearson's Chi squared test for categorical variables and Wald test p-values for coefficients in multinomial regression (i.e. log odds of each polydrug class) were derived. Multivariable models were adjusted for demographic (age, sex, education, ethnicity, income) and contextual variables (contact with NSP, city) based on a priori knowledge. The latent class with the largest membership was used as the referent category in order to maximise statistical power.

Multinomial logistic regression was performed using robust variance estimation to take into account the survey design, using the svy command in Stata version 13.1 [59]. The potential correlation of observations within the recruitment chains of RDS sampling was accounted for by clustering the standard errors within each recruitment seed [58]. RDS weights were not used as RDS weights did not influence weighted estimates when compared to unweighted estimates (Table S1) [60, 61]. Possible effect modification between behavioural risk and city were explored in multinomial regression. A complete case analysis was used and ten observations with missing data were disregarded. Models including biomarkers for $\mathrm{HCV}$ and $\mathrm{HSV}$ only included participants from Kohtla-Järve as these biomarkers were not collected in St Petersburg. RDS results are 
presented following guidelines outlined in STROBE-RDS [62].

\section{Ethics}

Ethical approval was obtained from the Ethics Review Board of the University of Tartu (Estonia), the Institutional Review Board at NGO Stellit in St. Petersburg (Russian Federation) and the Human Investigation Committee at Yale University (USA).

\section{Results}

Our study included 1402 active PWID who had injected drugs in the previous 4 weeks, were 18 years or older and lived in Kohtla-Järve $(\mathrm{n}=591)$ or St Petersburg ( $\mathrm{n}=811$ ). Sample characteristics were previously described [10, 16, 45-47] and are summarised in Table S1. RDS recruitment measures are shown in Table S2. Most PWID were male $(76 \%)$, of Russian ethnicity $(90 \%)$, had completed basic education (i.e. up to 9th grade) (68\%) and injected for over 5 years $(93 \%)$. Almost half had a nonregular income $(47 \%)$ and $38 \%$ were under 30 years old. Past month contact with an NSP was $43 \%$ with more PWID reporting contact in Kohtla-Järve than in St Petersburg (Table S1).

\section{Latent Class Model and Polydrug Use Class Membership}

Latent class models with 2, 3, 4, 5 and 6 classes were fitted to the data and fit statistics compared. Based on the different model fit indices and entropy, the five-class model was selected. Pearson's Chi squared and Likelihood ratio tests suggested a better fit for model 5 although AIC and BIC statistics were marginally lower for model 6 (Table S3).The 5-class model was preferred after examination of the 5-class and 6-class models. Both produced similar class assignments and the two very small classes in model 6 limited interpretation and subsequent analyses (not shown).

The largest class (class 5) included 56\% ( $\mathrm{n}=790)$ of all PWID and the sizes of the four remaining classes ranged from 97 to 217 injectors (Table 1). Table 1 shows the conditional probabilities of endorsing a drug variable for an individual classified in their most likely class in the fiveclass model. Most class-specific response probabilities for binary indicators were above 0.70 or below 0.30 , suggesting similar item responses for individuals in the same class and thus within-class homogeneity [52]. Drug use characteristics of each class in the 5-class model, including their qualitatively different primary (main) and additional drug class combinations are shown in Fig. 1.
The 124 PWID in class 1 had $56 \%$ probability of injecting a primary opiate drug and high probabilities of all other polydrug use variables (i.e. injection and use); a class referred to as "polydrug polyroute injection". The 97 class 2 PWID had high probabilities of (i) injecting opiates as main drug (94\%) and (ii) injecting additional stimulant(s) (100\%) and (iii) a null probability of using other drugs; this class was referred to as "opiate-stimulant polyinjection". Class 3 consisted of 174 PWID with similar probabilities of injecting opiates or ATS as primary drug ( $58 \%$ and $42 \%$, respectively), a high probability of using additional stimulants $(87 \%)$ and a null probability of injecting other drugs; class 3 was identified as "non-injection stimulant co-use". The 217 class 4 PWID, referred to as "opiate-opioid poly-injectors", were characterised by primary and secondary opiate/opioid injection (94\% and $100 \%$, respectively) and a null probability of using other drugs. Finally, class 5 PWID did not use or inject multiple drugs ("single drug injection") and had $87 \%$ probability of injecting an opiate as main drug and null probability on all other (poly)drug indicators.

The distribution of class membership varied between sites with "polydrug polyroute injection" (class 1) and "non-injection stimulant co-use" (class 3) injectors having a higher probability of being from Kohtla-Järve (66\% and $92 \%$, respectively); "opiate-stimulant poly-injection" (class 2) and “opiate-opioid poly-injection” (class 4) were more likely to be from St Petersburg (76\% and 94\%, respectively). Single drug injectors (class 5) had a higher probability of being from St Petersburg $(60 \%)$ than KohtlaJärve (40\%).

\section{Correlates of Polydrug Class Membership}

Multinomial univariate comparisons of socio-demographic characteristics, injecting and sexual risk behaviours, HIV and HCV prevalence between polydrug classes are shown in Table 2. Polydrug classes differed significantly from the sample average (in 17 of 23 variables) and among them, non-injection stimulant co-users (class 3) differed most often in four demographic, seven drug risk and three sexual risk variables. Opiate-stimulant poly-injectors (class 2) and opiate-opioid poly-injectors (class 4) reported higher injecting and sexual risk behaviours than single-drug injectors but did not differ on demographic characteristics. Polydrug polyroute injectors (class 1) differed on two demographic and two injecting behaviours.

More polydrug polyroute injectors (class 1) were younger than 30 years and of non-Russian ethnicity than single-drug injectors (class 5), injected more frequently and intensely. After adjusting for age, sex, education, income, ethnicity, contact with NSP and city in multivariable analysis, polydrug polyroute injectors had statistically 
Table 1 Conditional probabilities of drug use characteristics by class membership (5-class model)

\begin{tabular}{|c|c|c|c|c|c|c|c|c|c|c|c|c|}
\hline \multirow{2}{*}{$\begin{array}{l}\text { Latent class model } \\
\text { Class probabilities }\end{array}$} & \multicolumn{2}{|c|}{$\begin{array}{l}\text { Class } 1 \\
\text { Polydrug poly- } \\
\text { route injection } \\
(\%, n)\end{array}$} & \multicolumn{2}{|c|}{$\begin{array}{l}\text { Class } 2 \text { Opiate- } \\
\text { stimulant poly- } \\
\text { injection }(\%, n)\end{array}$} & \multicolumn{2}{|c|}{$\begin{array}{l}\text { Class } 3 \text { Non- } \\
\text { injection } \\
\text { stimulant co- } \\
\text { use }(\%, n)\end{array}$} & \multicolumn{2}{|c|}{$\begin{array}{l}\text { Class } 4 \text { Opiate- } \\
\text { opioid poly- } \\
\text { injection }(\%, \mathrm{n})\end{array}$} & \multicolumn{2}{|c|}{$\begin{array}{l}\text { Class } 5 \\
\text { Single drug } \\
\text { injection } \\
(\%, \mathrm{n})\end{array}$} & \multicolumn{2}{|c|}{$\begin{array}{l}\text { All classes } \\
(\%, \mathrm{n})\end{array}$} \\
\hline & $9 \%$ & 124 & $7 \%$ & 97 & $12 \%$ & 174 & $16 \%$ & 217 & $56 \%$ & 790 & $100 \%$ & 1402 \\
\hline \multicolumn{13}{|l|}{ Primary drug injected ${ }^{\mathrm{a}}$} \\
\hline Primarily injected opiates ${ }^{\mathrm{b}}$ & $56 \%$ & 69 & $94 \%$ & 91 & $58 \%$ & 102 & $93 \%$ & 201 & $87 \%$ & 689 & $82 \%$ & 1152 \\
\hline Primarily injected ATS & $27 \%$ & 33 & $4 \%$ & 4 & $42 \%$ & 73 & $6 \%$ & 13 & $13 \%$ & 102 & $16 \%$ & 223 \\
\hline No primary drug & $17 \%$ & 22 & $2 \%$ & 2 & $0 \%$ & 0 & $1 \%$ & 3 & $0 \%$ & 0 & $2 \%$ & 27 \\
\hline \multicolumn{13}{|l|}{ Other drugs and route of administration } \\
\hline Injected other opiate/opioid & $100 \%$ & 41 & $54 \%$ & 52 & $0 \%$ & 0 & $100 \%$ & 217 & $0 \%$ & 0 & $23 \%$ & 310 \\
\hline Injected other stimulant $(s)^{\mathrm{c}}$ & $100 \%$ & 107 & $100 \%$ & 97 & $0 \%$ & 0 & $0 \%$ & 0 & $0 \%$ & 0 & $15 \%$ & 204 \\
\hline Other opiate/opioid (non-injection) ${ }^{\mathrm{d}}$ & $100 \%$ & 52 & $0 \%$ & 0 & $34 \%$ & 59 & $0 \%$ & 0 & $0 \%$ & 0 & $8 \%$ & 111 \\
\hline Other stimulant (non-injection) & $100 \%$ & 104 & $0 \%$ & 0 & $87 \%$ & 151 & $0 \%$ & 0 & $0 \%$ & 0 & $19 \%$ & 255 \\
\hline \multicolumn{13}{|l|}{ Number other drug(s) injected } \\
\hline One & $64 \%$ & 79 & $46 \%$ & 45 & $0 \%$ & 0 & $97 \%$ & 211 & $0 \%$ & 0 & $24 \%$ & 335 \\
\hline Two & $30 \%$ & 37 & $45 \%$ & 44 & $0 \%$ & 0 & $3 \%$ & 6 & $0 \%$ & 0 & $6 \%$ & 87 \\
\hline Three & $6 \%$ & 8 & $8 \%$ & 8 & $0 \%$ & 0 & $0 \%$ & 0 & $0 \%$ & 0 & $1 \%$ & 16 \\
\hline \multicolumn{13}{|l|}{ City $^{a}$} \\
\hline St Petersburg & $34 \%$ & 42 & $76 \%$ & 74 & $8 \%$ & 14 & $94 \%$ & 205 & $60 \%$ & 476 & $58 \%$ & 811 \\
\hline Kohtla-Järve & $66 \%$ & 82 & $24 \%$ & 23 & $92 \%$ & 160 & $6 \%$ & 12 & $40 \%$ & 314 & $42 \%$ & 591 \\
\hline
\end{tabular}

Conditional probabilities are the probability that a PWID is a polydrug user, conditional on their answer "yes" to a specific drug question (i.e. observed variable). Conditional probabilities are graphed in Fig. 1 for each class of the five-class model

ATS Amphetamine-Type Stimulants

${ }^{a}$ Column totals

b Heroin/synthetic opioids

c Other stimulants included methamphetamines, ecstasy, cocaine and ketamine

d Non-injected use may be smoked, snorted or ingested in tablet or liquid form

Fig. 1 Polydrug use profiles for five-class solution of Latent Class model among PWID. The estimated probabilities for past month drug use are graphed based on latent class (C1-C5) membership shown in Table 1. The main axis $(0-100 \%)$ shows the probability of past month use for each drug variable. For example opiate-stimulant injectors $(\mathrm{C} 2)$ had a high probability of injecting an opiate as main drug $(96 \%)$, medium probability of injecting another opiate (54\%) and $100 \%$ probability of also injecting stimulants. The five binary variables only are shown for clarity (not shown are the variables for "number of drugs injected" and "number of noninjection drugs used")

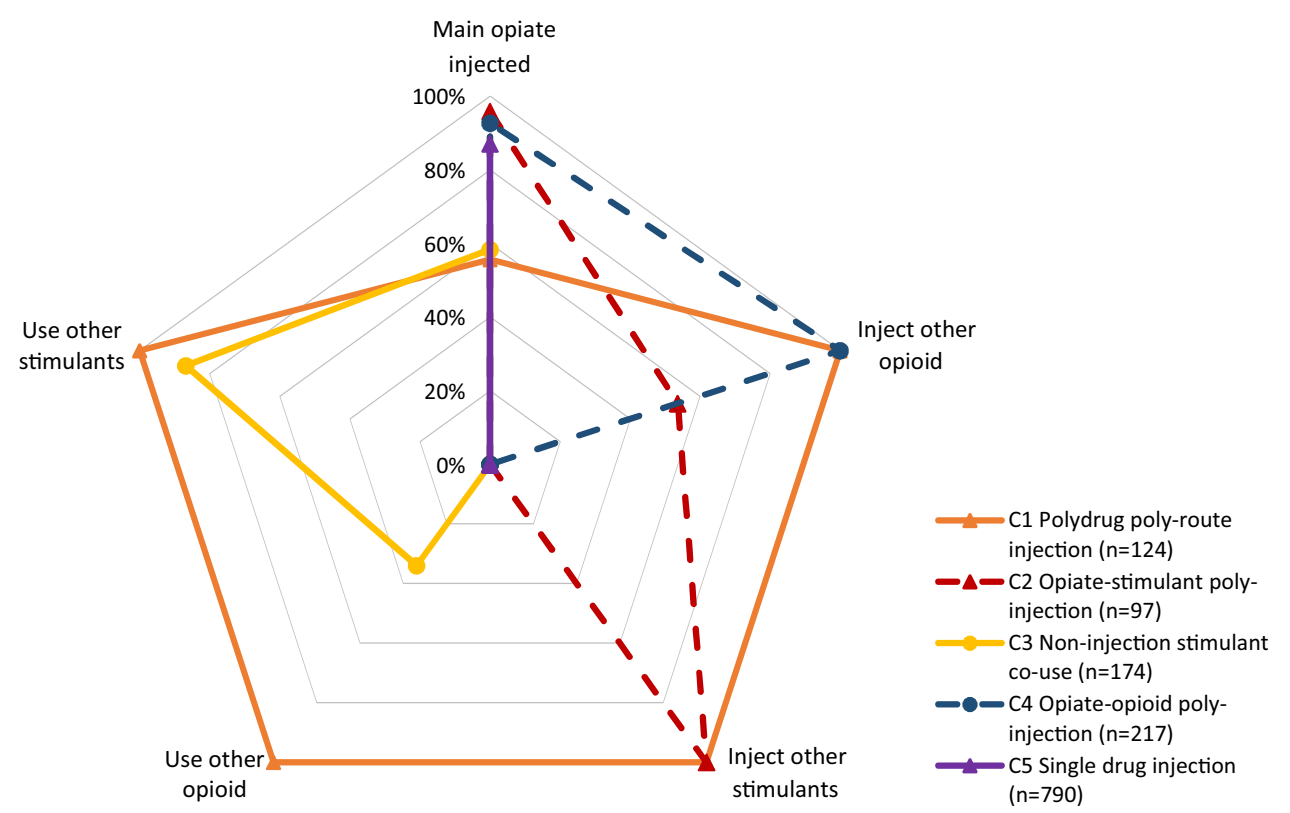


Table 2 Univariate comparisons of socio-demographics, service characteristics and HIV risk behaviours across latent classes

\begin{tabular}{|c|c|c|c|c|c|c|c|c|c|c|c|c|c|c|}
\hline & \multicolumn{2}{|c|}{$\begin{array}{l}\text { All PWID } \\
(\mathrm{n}=1402)^{\mathrm{a}}\end{array}$} & \multicolumn{2}{|c|}{$\begin{array}{l}\text { Class } 1 \\
\text { Polydrug } \\
\text { poly-route } \\
\text { injection } \\
(\mathrm{n}=124)\end{array}$} & \multicolumn{2}{|c|}{$\begin{array}{l}\text { Class } 2 \\
\text { Opiate- } \\
\text { stimulant } \\
\text { poly- } \\
\text { injection } \\
(\mathrm{n}=97)\end{array}$} & \multicolumn{2}{|c|}{$\begin{array}{l}\text { Class } 3 \\
\text { Non- } \\
\text { injection } \\
\text { stimulant } \\
\text { co-use } \\
(\mathrm{n}=174)\end{array}$} & \multicolumn{2}{|c|}{$\begin{array}{l}\text { Class } 4 \\
\text { Opiate- } \\
\text { opioid } \\
\text { poly- } \\
\text { injection } \\
(\mathrm{n}=217)\end{array}$} & \multicolumn{2}{|c|}{$\begin{array}{l}\text { Class } 5 \\
\text { Single } \\
\text { drug } \\
\text { injection } \\
(\mathrm{n}=790)\end{array}$} & $\begin{array}{l}\text { Pearson's } \\
X^{2}\end{array}$ & $\begin{array}{l}X^{2} \\
p_{-v a l u e}{ }^{b}\end{array}$ \\
\hline \multicolumn{15}{|l|}{ Demographic characteristics } \\
\hline Female gender & $24 \%$ & 335 & $28 \%$ & 35 & $23 \%$ & 22 & $31 \%$ & 54 & $21 \%$ & 45 & $23 \%$ & 179 & 8.26 & 0.082 \\
\hline Age $<30$ years & $38 \%$ & 535 & $45 \%{ }^{c}$ & 56 & $41 \%$ & 40 & $52 \%{ }^{\mathrm{c}}$ & 90 & $36 \%$ & 77 & $34 \%$ & 272 & 21.8 & 0.000 \\
\hline $\begin{array}{l}\text { Completed secondary } \\
\text { school }\end{array}$ & $26 \%$ & 359 & $27 \%$ & 34 & $26 \%$ & 25 & $25 \%^{\mathrm{c}}$ & 43 & $22 \%$ & 47 & $27 \%$ & 210 & 19.4 & 0.013 \\
\hline Completed higher education & $7 \%$ & 96 & $3 \%$ & 4 & $6 \%$ & 6 & $1 \%$ & 2 & $11 \%$ & 23 & $8 \%$ & 61 & & \\
\hline Non-Russian & $10 \%$ & 145 & $19 \%{ }^{c}$ & 23 & $5 \%$ & 5 & $17 \% \%^{\mathrm{c}}$ & 30 & $7 \%$ & 14 & $9 \%$ & 73 & 25.3 & 0.000 \\
\hline Non-regular income & $47 \%$ & 657 & $48 \%$ & 59 & $46 \%$ & 45 & $29 \%{ }^{c}$ & 49 & $48 \%$ & 105 & $51 \%$ & 399 & 28.5 & 0.000 \\
\hline City (Kohtla-Järve) & $42 \%$ & 591 & $66 \%$ & 82 & $24 \%$ & 23 & $92 \%$ & 160 & $6 \%$ & 12 & $40 \%$ & 314 & 340.9 & 0.000 \\
\hline \multicolumn{15}{|l|}{ Service characteristics ${ }^{\mathrm{d}}$} \\
\hline Drug/substitution treatment & $12 \%$ & 161 & $5 \%$ & 6 & $10 \%$ & 10 & $15 \%$ & 26 & $12 \%$ & 25 & $12 \%$ & 94 & 7.7 & 0.103 \\
\hline Contact with NSP & $43 \%$ & 570 & $54 \%$ & 62 & $28 \%{ }^{c}$ & 26 & $77 \% c$ & 125 & $19 \%$ & 40 & $44 \%$ & 317 & 143.9 & 0.000 \\
\hline \multicolumn{15}{|c|}{ Injecting risk behaviours (last month) } \\
\hline Injecting $<5$ years & $7 \%$ & 96 & $11 \%{ }^{c}$ & 13 & $4 \%$ & 4 & $14 \% \%^{c}$ & 24 & $2 \%$ & 5 & $6 \%$ & 50 & 24.2 & 0.000 \\
\hline Injecting daily or more & $31 \%$ & 427 & $42 \%$ & 52 & $53 \%{ }^{c}$ & 51 & $20 \%{ }^{c}$ & 34 & $37 \%{ }^{c}$ & 81 & $27 \%$ & 209 & 50.7 & 0.000 \\
\hline Injected $\geq$ twice a day & $46 \%$ & 642 & $61 \%{ }^{c}$ & 76 & $70 \%{ }^{c}$ & 68 & $43 \%$ & 74 & $50 \%{ }^{c}$ & 107 & $40 \%$ & 317 & 47.1 & 0.000 \\
\hline Shared needles/syringes & $36 \%$ & 502 & $31 \%$ & 38 & $55 \%{ }^{c}$ & 53 & $11 \%{ }^{\mathrm{c}}$ & 19 & $57 \% \%^{c}$ & 122 & $34 \%$ & 270 & 105.3 & 0.000 \\
\hline Lent needles/syringes & $37 \%$ & 520 & $32 \%$ & 39 & $57 \% \%^{c}$ & 55 & $15 \%{ }^{c}$ & 25 & $53 \%{ }^{c}$ & 114 & $37 \%$ & 287 & 78.3 & 0.000 \\
\hline Shared drug paraphernalia & $42 \%$ & 594 & $35 \%$ & 43 & $58 \%{ }^{c}$ & 56 & $16 \%{ }^{c}$ & 27 & $64 \% \%^{c}$ & 139 & $42 \%$ & 329 & 105.7 & 0.000 \\
\hline Filled from working syringe & $33 \%$ & 462 & $33 \%$ & 41 & $47 \% c$ & 46 & $13 \% \mathrm{c}$ & 23 & $40 \%$ & 86 & $34 \%$ & 266 & 44.4 & 0.000 \\
\hline \multicolumn{15}{|c|}{ Sexual risk behaviours (last 6 months) } \\
\hline Any sex in last 6 months & $78 \%$ & 1092 & $81 \%$ & 100 & $87 \% \%^{c}$ & 84 & $85 \% c$ & 146 & $74 \%$ & 160 & $76 \%$ & 602 & 13.0 & 0.011 \\
\hline$\geq 2$ sex partners & $43 \%$ & 447 & $42 \%$ & 36 & $54 \%{ }^{c}$ & 45 & $32 \% \mathrm{c}$ & 43 & $54 \%{ }^{c}$ & 87 & $41 \%$ & 236 & 20.8 & 0.000 \\
\hline Regular sex partner injects & $56 \%$ & 438 & $60 \%$ & 42 & $67 \%$ & 33 & $57 \% c$ & 70 & $73 \% \%^{c}$ & 63 & $51 \%$ & 230 & 18.3 & 0.001 \\
\hline Casual sex partner injects & $58 \%$ & 218 & $63 \%$ & 27 & $66 \%$ & 27 & $52 \%$ & 24 & $78 \% c$ & 46 & $50 \%$ & 94 & 16.3 & 0.003 \\
\hline Ever paid for sex & $5 \%$ & 54 & $9 \%$ & 8 & $4 \%$ & 3 & $6 \%$ & 8 & $2 \%$ & 3 & $6 \%$ & 32 & 4.7 & 0.319 \\
\hline \multicolumn{15}{|l|}{ Serological markers ${ }^{\mathrm{e}}$} \\
\hline HIV test positive & $58 \%$ & 818 & $53 \%$ & 66 & $61 \%$ & 59 & $60 \%$ & 105 & $59 \%$ & 127 & $58 \%$ & 461 & 1.87 & 0.759 \\
\hline $\mathrm{HCV}$ reactive & $74 \%$ & 441 & $72 \%$ & 59 & $65 \%$ & 15 & $73 \%$ & 116 & $75 \%$ & 9 & $77 \%$ & 242 & 2.75 & 0.599 \\
\hline HSV positive & $32 \%$ & 185 & $31 \%$ & 24 & $39 \%$ & 9 & $27 \%$ & 43 & $50 \%$ & 6 & $34 \%$ & 103 & 4.28 & 0.368 \\
\hline
\end{tabular}

${ }^{a}$ Column percentage

b Chi-square test

${ }^{\mathrm{c}}$ Unadjusted multinomial regression coefficient $\mathrm{p}$-values (statistically significant results $(p<0.05)$ are bolded). Pairwise comparisons using Class 5 as reference category

${ }^{\mathrm{d}}$ Drug/substitution treatment in past 12 months refers to drug substitution in Kohtla-Järve and any treatment in St Petersburg. Needle/syringe programme (NSP) contact in last 6 weeks

e Serological markers for hepatitis C (HCV) and herpes simplex virus (HSV) were available for Kohtla-Järve only

significantly higher odds of injecting more frequently and intensely, sharing needles and syringes, drug paraphernalia and back-loading (filling a syringe from a pre-filled syringe) than single-drug injectors (Table 3 ).

Opiate-stimulant poly-injectors (class 2) had lower contact with an NSP, they reported more frequent injecting, greater injection intensity, more sharing and lending of needles and syringes, and back-loading in the past month than single-drug injectors (Table 2). More PWID in this class also reported multiple sex partners. After adjustment in multivariable analyses, opiate-stimulant poly-injection remained positively associated with frequent and intense injecting, sharing needles/syringes, sharing drug paraphernalia and back-loading compared to single drug 
Table 3 Adjusted multinomial analysis of demographic, injecting and sexual risk behaviours, and serological markers of infections with poly(drug) use latent class membership

\begin{tabular}{|c|c|c|c|c|}
\hline \multirow[b]{2}{*}{$\begin{array}{l}\text { Reference group: Class } 5 \text { single } \\
\text { drug injection }\end{array}$} & \multicolumn{4}{|c|}{ Adjusted odds ratios $(95 \% \mathrm{CI})$} \\
\hline & $\begin{array}{l}\text { Class } 1 \text { Polydrug } \\
\text { polyroute injection }\end{array}$ & $\begin{array}{l}\text { Class } 2 \text { Opiate-stimulant } \\
\text { poly-injection }\end{array}$ & $\begin{array}{l}\text { Class } 3 \text { Non-injection } \\
\text { stimulant co-use }\end{array}$ & $\begin{array}{l}\text { Class } 4 \text { Opiate-opioid } \\
\text { poly-injection }\end{array}$ \\
\hline \multicolumn{5}{|l|}{ Demographic and services } \\
\hline Female gender & $1.1(0.7-2.0)$ & $0.8(0.4-1.7)$ & $1.4(0.9-2.1)$ & $0.8(0.5-1.2)$ \\
\hline Age $<30$ & $1.3(0.9-1.8)$ & $1.7(1.0-2.9)$ & $1.5(1.0-2.2)$ & $1.6(1.1-2.3)^{\mathrm{a}}$ \\
\hline Non-Russian & $1.8(1.1-3.1)^{\mathrm{a}}$ & $0.8(0.3-2.1)$ & $1.1(0.6-1.9)$ & $1.6(1.1-2.4)^{\mathrm{a}}$ \\
\hline Completed secondary school & $0.8(0.5-1.3)$ & $0.9(0.5-1.6)$ & $0.7(0.5-1.0)^{\mathrm{c}}$ & $0.9(0.5-1.5)$ \\
\hline Non-regular income & $1.0(0.7-1.6)$ & $0.7(0.4-1.2)$ & $0.6(0.5-0.7)^{\mathrm{c}}$ & $0.8(0.5-1.1)$ \\
\hline City (Kohtla-Järve) & $3.4(1.7-6.6)^{\mathrm{a}}$ & $0.5(0.3-1.0)$ & $14.9(7.8-28.9)^{\mathrm{c}}$ & $0.1(0.1-0.2)^{\mathrm{a}}$ \\
\hline \multicolumn{5}{|l|}{ Service characteristics ${ }^{\mathrm{b}}$} \\
\hline $\begin{array}{l}\text { Contact with NSP (last } \\
6 \text { weeks) }\end{array}$ & $0.7(0.4-1.3)$ & $0.7(0.4-1.3)$ & $1.0(0.6-1.6)$ & $0.9(0.6-1.3)$ \\
\hline $\begin{array}{l}\text { Drug treatment (last } \\
12 \text { months) }\end{array}$ & $0.3(0.1-1.5)$ & $0.4(0.2-1.6)$ & $0.7(0.3-1.5)$ & $0.7(0.2-2.3)$ \\
\hline \multicolumn{5}{|c|}{ Injecting risk behaviours (last month) } \\
\hline Injecting $<5$ years & $1.1(0.7-1.9)$ & $1.6(0.4-6.3)$ & $0.7(0.4-1.3)$ & $1.8(0.5-5.9)$ \\
\hline Injected daily or more & $2.5(1.1-5.7)^{\mathrm{a}}$ & $3.0(1.5-5.8)^{\mathrm{a}}$ & $0.9(0.5-1.5)$ & $1.3(1.0-1.8)$ \\
\hline Injected $\geq$ twice a day & $2.7(1.3-5.9)^{\mathrm{a}}$ & $4.0(2.3-6.9)^{\mathrm{a}}$ & $1.1(0.8-1.6)$ & $1.6(1.1-2.4)^{\mathrm{a}}$ \\
\hline Shared needles/syringes & $2.5(1.3-4.8)^{\mathrm{a}}$ & $2.3(1.7-3.2)^{\mathrm{a}}$ & $1.6(0.7-4.0)$ & $1.6(1.1-2.4)^{\mathrm{a}}$ \\
\hline Lent needles/syringes & $1.0(0.5-1.9)$ & $2.4(1.4-3.9)^{\mathrm{a}}$ & $0.9(0.5-1.7)$ & $1.4(0.9-1.9)$ \\
\hline Shared paraphernalia & $2.7(1.4-4.9)^{\mathrm{a}}$ & $1.8(1.2-2.7)^{\mathrm{a}}$ & $2.4(0.9-6.9)$ & $1.2(0.9-1.7)$ \\
\hline Filled from working syringe & $3.6(2.3-5.8)^{\mathrm{a}}$ & $1.8(1.1-3.1)^{\mathrm{a}}$ & $3.2(1.4-7.2)^{\mathrm{a}}$ & $0.7(0.5-1.1)$ \\
\hline \multicolumn{5}{|c|}{ Sexual risk behaviours (last 6 months) } \\
\hline Any sex in last 6 months & $1.1(0.6-2.2)$ & $1.9(1.1-3.5)^{\mathrm{a}}$ & $1.5(1.1-2.2)^{\mathrm{a}}$ & $0.9(0.6-1.1)$ \\
\hline$\geq 2$ sex partners & $1.2(0.8-1.8)$ & $1.7(1.2-2.4)^{\mathrm{a}}$ & $1.1(0.8-1.5)$ & $1.6(1.2-2.1)^{\mathrm{a}}$ \\
\hline Regular sex partner injects & $1.5(0.8-3.2)$ & $1.9(0.8-4.4)$ & $1.2(0.9-1.6)$ & $3.2(2.1-4.9)^{\mathrm{a}}$ \\
\hline Casual sex partner injects & $1.7(1.0-2.9)$ & $1.3(0.5-3.5)$ & $1.1(0.5-2.6)$ & $2.1(1.1-3.9)^{\mathrm{a}}$ \\
\hline Ever paid for sex & $1.0(0.4-2.4)$ & $0.6(0.2-1.7)$ & $0.5(0.2-1.2)$ & $0.5(0.1-1.9)$ \\
\hline \multicolumn{5}{|l|}{ Serological markers ${ }^{\mathrm{c}}$} \\
\hline HIV test positive & $0.7(0.4-1.3)$ & $1.1(0.7-1.8)$ & $0.9(0.7-1.3)$ & $1.3(0.9-1.8)$ \\
\hline $\mathrm{HCV}$ reactive & $0.7(0.2-2.3)$ & $0.6(0.1-2.9)$ & $0.8(0.5-1.3)$ & $0.8(0.2-4.2)$ \\
\hline HSV positive & $0.6(0.3-1.1)$ & $1.1(0.5-2.8)$ & $0.6(0.4-1.0)$ & $2.0(0.6-6.6)$ \\
\hline
\end{tabular}

Multivariable multinomial regression models adjusted for age, sex, education, income, ethnicity, contact with needle and syringe programme and city (drug/substitution treatment did not differ significantly across classes)

CI confidence intervals, NSP needle and syringe programme

${ }^{\text {a }}$ Regression coefficient $p$ value $\leq 0.05$

b Drug/substitution treatment in past 12 months refers to drug substitution in Kohtla-Järve and any treatment in St Petersburg

c Serological markers for hepatitis C (HCV) and herpes simplex virus (HSV) were available for Kohtla-Järve only

injection (Table 3). Opiate-stimulant poly-injectors were also more likely to report any sex in the past 6 months and multiple sex partners than single-drug injectors.

More non-injection stimulant co-users (class 3) were under 30 years of age, of non-Russian ethnicity and from Kohtla-Järve and fewer reported an irregular source of income compared to single-drug injectors (Table 2). Noninjection stimulant co-users reported lower injection risk behaviours but more sex in the past 6 months compared to single-drug injectors and more PWID in this class had a regular partner who injected drugs. In multivariable analysis, non-injection stimulant co-users, who were less likely to have completed secondary education, to have a regular income and to be from Kohtla-Järve, reported generally lower injecting risks than single-drug injectors (Table 3). Non-injection stimulant co-users had greater odds of backloading and were more likely to have had sex in the last 6 months compared to single-drug injectors. 
The 217 opiate-opioid poly-injectors (class 4) reported more sharing of needles and syringes and more sharing of drug paraphernalia than single-drug injectors in unadjusted analysis (Table 2). More PWID in this class reported multiple sex partners and a sex partner who injected drugs compared to single-drug injectors. In multivariable analyses, opiate-opioid poly-injectors had higher odds of being younger than 30 years old, non-Russian and from St Petersburg (Table 3). Opiate-opioid poly-injectors had greater odds of injecting more intensely and of sharing needles/syringes, compared to single-drug injectors. They were also more likely to have multiple sex partners and a sex partner who injected drugs.

Despite significant differences in injecting and sexual risk behaviours, no statistically significant differences were found in HIV and HCV prevalence when any of the polydrug classes were compared to single drug-injectors, who were mainly opiate injectors. Differences in the prevalence of HIV or HCV among classes may emerge using another reference group. For example, opiate-opioid poly-injectors were more likely to be HIV positive compared to polydrug-polyroute injectors (adjusted OR 1.8; 95\% confidence interval (CI) 1.1-3.2).

Potential effect modification between behavioural risk and city, examined in multinomial regressions, suggested that odds ratios were consistent for sexual and injecting risk behaviours, except for injecting frequency. The odds of frequent injecting remained significantly higher for poly-injectors (compared to single-drug injectors) in $\mathrm{St}$ Petersburg but was not significant in Kohtla-Järve in models examining effect modification (Table S4).

\section{Discussion}

Our study found that polydrug use was substantial among PWID in Kohtla-Järve and St Petersburg, 44\% of whom belonged to one of four polydrug classes. It also uncovered considerable differences in HIV risk behaviours with significantly greater injecting and sexual risk among polydrug than single-drug injectors (Table 4). Despite non-significant differences in $\mathrm{HIV}$ and $\mathrm{HCV}$ prevalence, riskier behaviours found among polydrug injectors suggest increased potential for continuing transmission of blood borne and sexually transmitted infections. Among polydrug classes, polydrug-polyroute injectors engaged in more frequent injecting and sharing risk behaviours than exclusive injectors. However, opiate-stimulant poly-injectors and opiate-opioid poly-injectors both reported more injecting and sexual risk behaviours than single drug injectors, with opiate-opioid poly-injectors also reporting sex partners who injected drugs. Non-injection stimulant co-users differed less from single-drug injectors but were more likely to back-load syringes and have had sex in the last six months (Table 4).

Our findings that opiate-stimulant poly-injection was associated with frequent and intense injecting, needle/syringe sharing and multiple sex partners were consistent with several studies where PWID injecting opiates and stimulants (methamphetamines or cocaine) were more likely to report greater injection risks (i.e. sharing and lending needles/syringes [41, 63-66] and more daily injections [39-41, 66-69]) than single opiate injectors [40, 54, 68].

That opiate-opioid poly-injection, more likely to be found in St Petersburg, was associated with more daily injections and needle/syringe sharing in our study, likely reflected the localized availability and co-injection of illicit methadone and heroin in this city [45]. Injecting synthetic opioids and heroin has been associated with greater injection risks in some settings, including needle/syringe-sharing and more injections per day [70-73].

The following strengths and limitations are acknowledged. The samples may not be representative of all PWID in the two cities as they relied on a chain referral sampling methodology. RDS has nevertheless been an effective method to recruit hard-to-reach sub-groups, within key populations at risk of HIV infection, not reached by programmes [74].

Possible under-reporting of stigmatised behaviours in PWID self-reports and bias towards socially desirable answers were minimised by ensuring confidentiality during face-to-face interviews, in settings that were safe and familiar to PWID. Further, comparisons of missing data revealed no differences between classes suggesting that if bias due to non-response were present, it would affect polydrug sub-groups similarly.

It is also possible that short-term drug use patterns measured over four-weeks may not be a good predictor of HIV and HCV prevalence as polydrug class membership (and their associated behaviours) may not necessarily be longitudinally consistent or may not reflect cumulative exposure to certain risk factors. This may explain that, despite sexual and injecting risk behaviours being associated with polydrug use classes, we did not detect any association with HIV or HCV prevalence. Longitudinal studies among PWID may contribute to establish whether polydrug practices evolve over time, and especially how they might change over the course of a drug injection "career", or as a function of the drug environment [53, 75].

The strengths of our analyses were the large sample size and combination of two similar samples of PWID, surveyed using comparable methods, instruments and tools, which could increase the generalisability of our results. Although the drug use and HIV epidemics were similar enough for surveys to be pooled, potential unobserved 
Table 4 Summary of associations between demographic, injecting and sexual risk behaviours and latent poly(drug) use classes as compared to single drug injectors

\begin{tabular}{|c|c|c|c|}
\hline $\begin{array}{l}\text { Class } 1 \text { Polydrug polyroute } \\
\text { injection }\end{array}$ & $\begin{array}{l}\text { Class } 2 \text { Opiate-stimulant poly- } \\
\text { injection }\end{array}$ & $\begin{array}{l}\text { Class } 3 \text { Non-injection stimulant co- } \\
\text { use }\end{array}$ & $\begin{array}{l}\text { Class } 4 \text { Opiate-opioid poly- } \\
\text { injection }\end{array}$ \\
\hline- & $\uparrow<30$ years old & $\uparrow<30$ years old & $\uparrow<30$ years old \\
\hline$\uparrow$ non- Russian ethnicity & - & - & $\uparrow$ non- Russian ethnicity \\
\hline- & - & $\downarrow$ Secondary education & - \\
\hline- & - & $\uparrow$ Non-regular income & - \\
\hline- & - & - & - \\
\hline$\uparrow$ Kohtla-Järve & - & $\uparrow$ Kohtla-Järve & $\uparrow$ St Petersburg \\
\hline$\uparrow$ Frequent injecting & $\uparrow$ Frequent injecting & - & $=$ Frequent injecting \\
\hline$\uparrow$ Intense injecting & $\uparrow$ Intense injecting & - & $\uparrow$ Intense injecting \\
\hline$\uparrow$ Shared needles/syringes & $\uparrow$ Shared needles/syringes & - & $\uparrow$ Shared needles/syringes \\
\hline$\uparrow$ Sharing paraphernalia & $\uparrow$ Sharing paraphernalia & - & - \\
\hline$\uparrow$ Back-loaded & $\uparrow$ Back-loaded & $\uparrow$ Back-loaded & - \\
\hline- & $\uparrow$ Any sex last 6 months & $\uparrow$ Any sex last 6 months & - \\
\hline- & $\uparrow$ Multiple sex partners & - & $\uparrow$ Multiple sex partners \\
\hline- & - & - & $\uparrow$ Regular sex partner injects \\
\hline- & - & - & $\uparrow$ Casual sex partner injects \\
\hline
\end{tabular}

“ $\uparrow$ and $\downarrow "$ indicate positive and negative associations, respectively. "=” positive direction but non-significant association

differences and within-survey correlations were possible and accounted for in the analysis. Our examination of effect modifications suggested that associations between polydrug use and risk behaviours were similar for both cities though the magnitude of some associations was greater for St Petersburg than Kohtla-Järve. Important structural differences in the two cities may also account for such differences. For instance, parenteral and oral routes of opioid use may co-occur in Kohtla-Järve, where oral methadone treatment is available but often at low doses; opioid highs may be sought by injecting locally available (methyl)-fentanyl. In St. Petersburg, however, heroin and illicit methadone are both injected and likely contribute most cases of opioid poly-injection.

The sizeable heterogeneity in polydrug use patterns and HIV risk behaviours among PWID highlight the need to expand opiate substitution treatment following evidencebased dosage guidelines at the public health scale. HIV prevention and treatment interventions need to be tailored to the risk profiles and drug combinations injected by PWID. Our findings also emphasize the importance of ongoing and continuous drug monitoring among PWID, among whom polydrug use is frequent and may provide a marker of risk behaviours and HIV/HCV risk [76].

In these settings, HIV incidence among PWID was as high as 14.1 (95\% CI 10.7-17.6) HIV infections per 100 person-years in St Petersburg in 2008 and 9 HIV infections per 100 person-years in Tallinn in 2009 [6, 9, 13, 77, 78]. Monitoring drugs and combinations commonly used by PWID could enable programmes to deliver appropriate injecting and sexual risk reduction messages, sufficient supplies of clean injection equipment and promotion of safe sexual behaviours with all sex partners. Whereas opiate substitution modalities should be expanded (or legalised in the case of Russia) to reduce risk among opiate-opioid poly-injectors, alternative drug treatment modalities are needed for opiate-stimulant poly-injectors. In settings where stimulant and opiates are injected, opiate substitution may not be effective and drug treatment alternatives including behavioural and pharmacological approaches for stimulant users are needed [27, 40, 79, 80]. Improving access to primary health care services should also be encouraged to provide an entry point for PWID with different drug use and risk profiles. Recognising and addressing polydrug use as drug combinations, in drug treatment and HIV treatment settings may also help to increase PWID retention, adherence to therapy and hence, improve treatment outcomes [24, 28, 81, 82].

Acknowledgements We are grateful to the field staff who conducted the surveys, staff and peers who assisted in identifying seeds and to the harm reduction programmes who provided interviewing facilities and serological testing ('Me aitamesind' in Kohtla-Järve and 'Diakonia' in St Petersburg).

Author contributions RH, OL and AU designed the original research and survey instruments, and led data collection in St Petersburg and Kohtla-Järve, respectively. ITE and MCB developed the research questions for this study. ITE and MMG conducted the statistical analyses, interpreted results and wrote the manuscript. All authors critically reviewed the manuscript.

\section{Compliance with ethical standards}

Conflict of interest The authors report no conflicts of interest. 
Ethical approval All procedures performed in the course of the study were in accordance with the ethical standards of the relevant institutional and/or national research committee and with the 1964 Helsinki declaration and its later amendments. Ethical approval was obtained from the Ethics Review Board of the University of Tartu (Estonia), the Institutional Review Board at NGO Stellit in St. Petersburg (Russian Federation) and the Human Investigation Committee at Yale University (USA).

Informed consent Informed consent was obtained from all individual participants included in the study.

Open Access This article is distributed under the terms of the Creative Commons Attribution 4.0 International License (http://crea tivecommons.org/licenses/by/4.0/), which permits unrestricted use, distribution, and reproduction in any medium, provided you give appropriate credit to the original author(s) and the source, provide a link to the Creative Commons license, and indicate if changes were made.

\section{References}

1. United Nations Joint Programme on AIDS. Global AIDS update. Geneva: United Nations Joint Programme on AIDS; 2016.

2. United Nations Office on Drugs and Crime. World Drug Report 2016. Vienna: UNODC, Research and Trend Analysis Branch; 2016. Contract No.: E.16.XI.7.

3. United Nations Office on Drugs and Crime. World Drug Report 2015. Vienna: UNODC, Research and Trend Analysis Branch; 2015. Contract No.: E.12.XI.1.

4. Walsh N, Maher L. HIV and HCV among people who inject drugs in Central Asia. Drug Alcohol Depend. 2013;132(SUPPL1): S37-40.

5. El-Bassel N, Strathdee SA, Sadr WME. HIV and people who use drugs in central Asia: confronting the perfect storm. Drug Alcohol Depend. 2013;132(SUPPL1):S2-6.

6. Jolley E, Rhodes T, Platt L, Hope V, Latypov A, Donoghoe M, et al. HIV among people who inject drugs in Central and Eastern Europe and Central Asia: a systematic review with implications for policy. BMJ Open. 2012;2(5):e001465.

7. National Institute for Health Development. HIV in Estonia: situation, prevention, treatment, and care. Tallinn: National Institute for Health Development, Development NIfH; 2016.

8. Mathers BM, Degenhardt L, Phillips B, Wiessing L, Hickman M, Strathdee SA, et al. Global epidemiology of injecting drug use and HIV among people who inject drugs: a systematic review. Lancet. 2008;372(9651):1733-45.

9. Uuskula A, Kals M, Rajaleid K, Abel K, Talu A, Ruutel K, et al. High-prevalence and high-estimated incidence of HIV infection among new injecting drug users in Estonia: need for large scale prevention programs. J Public Health. 2008;30(2):119-25.

10. Uuskula A, Raag M, Vorobjov S, Ruutel K, Lyubimova A, Levina OS, et al. Non-fatal overdoses and related risk factors among people who inject drugs in St. Petersburg, Russia and Kohtla-Jarve, Estonia. Bmc Public Health. 2015;15(1):1255.

11. Uuskula A, Raag M, Folch C, Prasad L, Karnite A, van Veen MG, et al. Self-reported testing, HIV status and associated risk behaviours among people who inject drugs in Europe: important differences between East and West. AIDS. 2014;28(11):1657-64.

12. Talu A, Rajaleid K, Abel-Ollo K, Ruutel K, Rahu M, Rhodes T, et al. HIV infection and risk behaviour of primary fentanyl and amphetamine injectors in Tallinn, Estonia: implications for intervention. Int J Drug Policy. 2010;21(1):56-63.
13. Vorobjov S, Des Jarlais DC, Abel-Ollo K, Talu A, Rüütel K, Uusküla A. Socio-demographic factors, health risks and harms associated with early initiation of injection among people who inject drugs in Tallinn, Estonia: evidence from cross-sectional surveys. Int J Drug Policy. 2013;24(2):150-5.

14. National Institute for Health Development. HIV and other infections and the prevalence of risk behaviours in drug addicts. National Institute for Health Development; 2008.

15. United Nations Office on Drugs and Crime. World drug report 2014. Vienna: UNODC, Research and Trend Analysis Branch; 2014

16. Eritsyan K, Heimer R, Barbour R, Odinokova V, White E, Rusakova MM, et al. Individual-level, network-level and citylevel factors associated with HIV prevalence among people who inject drugs in eight Russian cities: a cross-sectional study. BMJ Open. 2013;3(6):e002645.

17. United Nations Office on Drugs and Crime. World drug report 2012. Vienna: UNODC, Research and Trend Analysis Branch; 2012. Contract No.: E.12.XI.1.

18. Grund J-P, Zabransky T, Irwin K, Heimer R. Stimulant use in central and Eastern Europe. In: Pates R, Diane Riley, editors. Interventions for amphetamine misuse. Blackwell: Wiley; 2009.

19. Platt L, Bobrova N, Rhodes T, Uuskula A, Parry JV, Ruutel K, et al. High HIV prevalence among injecting drug users in Estonia: implications for understanding the risk environment. AIDS. 2006;20(16):2120-3.

20. European Monitoring Centre for Drugs and Drug Addiction. Amphetamine: a European Union perspective in the global context. Europol Trends Organ Crim. 2012;15(2-3):222-40.

21. European Monitoring Centre for Drugs and Drug Addiction. Problem amphetamine and methamphetamine use in Europe. EMCDDA; 2010. Report No.: ISBN/ISSN: 978-92-9168-450-2.

22. United Nations Office on Drugs and Crime. Trends and patterns of amphetamine-type stimulants and new psychoactive substances. Vienna: United Nations Office on Drugs and Crime, Global SMART Programme (East Asia); 2015.

23. Tavitian-Exley I, Maheu-Giroux M, Platt L, Heimer R, Uuskula A, Levina O, et al. Differences in risk behaviour and HIV status between people who inject amphetamines and opiates in Estonia and Russia. Under review.

24. European Monitoring Centre for Drugs and Drug Addiction. Polydrug use: patterns and responses. Lisbon: EMCDDA; 2009.

25. Darke S, Zador D. Fatal heroin 'overdose': a review. Addiction. 1996;91(12):1765-72.

26. Coffin PO, Galea S, Ahern J, Leon AC, Vlahov D, Tardiff K. Opiates, cocaine and alcohol combinations in accidental drug overdose deaths in New York City, 1990-1998. Addiction. 2003;98(6):739-47.

27. Williamson A, Darke S, Ross J, Teesson M. The association between cocaine use and short-term outcomes for the treatment of heroin dependence: findings from the Australian Treatment Outcome Study (ATOS). Drug Alcohol Rev. 2006;25(2):141-8.

28. Nosyk B, Min JE, Colley G, Lima VD, Yip B, Milloy MJS, et al. The causal effect of opioid substitution treatment on HAART medication refill adherence. AIDS. 2015;29(8):965-73.

29. Tavitian-Exley I, Vickerman P, Bastos FI, Boily M-C. Influence of different drugs on HIV risk in people who inject: systematic review and meta-analysis. Addiction. 2015;110(4):572-84.

30. Betts KS, Chan G, McIlwraith F, Dietze P, Whittaker E, Burns L, et al. Differences in polysubstance use patterns and drug-related outcomes between people who inject drugs receiving and not receiving opioid substitution therapies. Addiction. 2016;111(7): 1214-23.

31. Harrell PT, Fu H, et al. Commentary on Betts et al. (2016): polysubstance use and opioid substitution therapy among people who inject drugs. Addiction. 2016;111(7):1224-5. 
32. Schensul JJ, Convey M, Burkholder G. Challenges in measuring concurrency, agency and intentionality in polydrug research. Addict Behav. 2005;30(3):571-4.

33. Baker A, Kochan N, Dixon J, Wodak A, Heather N. Drug use and HIV risk-taking behaviour among injecting drug users not currently in treatment in Sydney, Australia. Drug Alcohol Depend. 1994;34(2):155-60.

34. Judd A, Hutchinson S, Wadd S, Hickman M, Taylor A, Jones S, et al. Prevalence of, and risk factors for, hepatitis $\mathrm{C}$ virus infection among recent initiates to injecting in London and Glasgow: cross sectional analysis. J Viral Hepat. 2005;12(6):655-62.

35. Folch C, Merono M, Casabona J. Factors associated with sharing syringes among street-recruited injecting drug users. [Spanish]. Med Clin (Barc). 2006;127(14):526-32.

36. Kedia S, Sell MA, Relyea G. Mono- versus polydrug abuse patterns among publicly funded clients. Subst Abuse Treat Prevent Policy. 2007;2:33.

37. McCutcheon AL. Latent class analysis. Newbury Park: Sage Publications; 1987.

38. Flaherty BP, Kiff CJ. Latent class and latent profile models. In: Cooper H, Camic PM, Long DL, Panter AT, Rindskopf D, Sher KJ, editors. APA handbook of research methods in psychology, Vol 3: Data analysis and research publication. Washington, DC: American Psychological Association; 2012. p. 391-404.

39. Harrell PT, Mancha BE, Petras H, Trenz RC, Latimer WW. Latent classes of heroin and cocaine users predict unique HIV/ HCV risk factors. Drug Alcohol Depend. 2012;122(3):220-7.

40. Roy E, Richer I, Arruda N, Vandermeerschen J, Bruneau J. Patterns of cocaine and opioid co-use and polyroutes of administration among street-based cocaine users in Montreal, Canada. Int J Drug Policy. 2013;24(2):142-9.

41. Meacham MC, Rudolph AE, Strathdee SA, Rusch ML, Brouwer $\mathrm{KC}$, Patterson TL, et al. Polydrug use and HIV risk among people who inject heroin in Tijuana, Mexico: a latent class analysis. Subst Use Misuse. 2015;50(10):1-9.

42. Heckathorn DD. Respondent-driven sampling: a new approach to the study of hidden populations. Soc Probl. 1997;44(2): 174-99.

43. Heckathorn DD, Semaan S, Broadhead RS, Hughes JJ. Extensions of respondent-driven sampling: a new approach to the study of injection drug users aged 18-25. AIDS Behav. 2002;6(1): 55-67.

44. Magnani R, Sabin K, Saidel T, Heckathorn D. Review of sampling hard-to-reach and hidden populations for HIV surveillance. AIDS. 2005;19(Suppl 2):S67-72.

45. Heimer R, Lyubimova A, Barbour R, Levina OS. Emergence of methadone as a street drug in St. Petersburg, Russia. Int J Drug Policy. 2015;27:97-104.

46. Dukhovlinova E, Masharsky A, Toussova O, Verevochkin S, Solovyeva T, Meringof M, et al. Two Independent HIV Epidemics in Saint Petersburg, Russia revealed by molecular epidemiology. AIDS Res Hum Retroviruses. 2015;31(6):608-14.

47. Cepeda JA, Niccolai LM, Lyubimova A, Kershaw T, Levina O, Heimer R. High-risk behaviors after release from incarceration among people who inject drugs in St. Petersburg, Russia. Drug Alcohol Depend. 2015;147:196-202.

48. Johnston LG, Sabin K. Sampling hard-to-reach populations with respondent driven sampling. Method Innov. Online. 2010;5(2): $38-48$.

49. Des Jarlais DC, Perlis TE, Stimson GV, Poznyak V, Collab WPIDI. Using standardized methods for research on HIV and injecting drug use in developing/transitional countries: case study from the WHO Drug Injection Study Phase II. BMC Public Health. 2006;6(1):54.

50. European Monitoring Centre for Drugs and Addiction. DRID guidance module: behavioural indicators for people who inject drugs. In: EMCDDA, editor. VERSION 1.0 ed: EMCDDA DRID Behavioural Indicators Module; 2014.

51. Hagenaars JA, McCutcheon AL. Applied latent class analysis. Cambridge: Cambridge University Press; 2002.

52. Roesch SC, Villodas M, Villodas F. Latent class/profile analysis in maltreatment research: a commentary on Nooner et al., Pears et al., and looking beyond. Child Abuse Negl. 2010;34(3): 155-60.

53. Lanza ST, Bray BC, Collins LM. An introduction to latent class and latent transition analysis. In: Schinka JA, Velicer WF, Weiner IB, editors. Handbook of psychology, vol. 2. 2nd ed., Research methods in psychologyHoboken: Wiley; 2013. p. 691-715.

54. Monga N, Rehm J, Fischer B, Brissette S, Bruneau J, El-Guebaly $\mathrm{N}$, et al. Using latent class analysis (LCA) to analyze patterns of drug use in a population of illegal opioid users. Drug Alcohol Depend. 2007;88(1):1-8.

55. Muthén LK, Muthén BO. Mplus User's Guide. Los Angeles: Muthén \& Muthén; 1998-2012.

56. Bengt OM. Latent variable modeling in heterogeneous populations. Psychometrika. 2011;54(4):557-85.

57. Hosmer DL, Lemeshow S, Sturdivant X. R. Applied logistic regression. New York: Wiley; 2013.

58. Kirkwood BS, Sterne JAC. Essential medical statistics. 2nd ed. Oxford: Wiley; 2003.

59. StataCorp. Stata survey data reference manual (Release 13). 2013. StataCorp LP. College Station, TX: StataCorp. Stata: Release 13. Available from: http://www.stata.com/manuals13/ svy.pdf.

60. Solon G, Haider S, Wooldridge J. What are we weighting for? (NBER Working Paper 18859). 2013.

61. Korn EL, Graubard BI. Epidemiologic studies utilizing surveys: accounting for the sampling design. Am J Public Health. 1991;81(9):1166-73.

62. White RG, Hakim AJ, Salganik MJ, Spiller MW, Johnston LG, Kerr L, et al. Strengthening the reporting of observational studies in epidemiology for respondent-driven sampling studies: "STROBE-RDS" statement. J Clin Epidemiol. 2015;68(12): 1463-71.

63. Hudgins R, McCusker J, Stoddard A. Cocaine use and risky injection and sexual behaviors. Drug Alcohol Depend. 1995;37(1): 7-14.

64. Gleghorn AA, Marx R, Vittinghoff E, Katz MH. Association between drug use patterns and HIV risks among homeless, runaway, and street youth in Northern California. Drug Alcohol Depend. 1998;51(3):219-27.

65. Shearer J, Johnston J, Fry CL, Kaye S, Dillon P, Dietze P, et al. Contemporary cocaine use patterns and associated harms in Melbourne and Sydney, Australia. Drug Alcohol Rev. 2007;26(5): 537-43.

66. Braine N, Des Jarlais DC, Goldblatt C, Zadoretzky C, Turner C. HIV risk behavior among amphetamine injectors at U.S. syringe exchange programs. AIDS Educ Prev. 2005;17(6):515-24.

67. Kral AH, Lorvick J, Martinez A, Lewis MA, Orr WA, Anderson $\mathrm{R}$, et al. HIV prevalence and risk among heterosexual methamphetamine injectors in California. Subst Use Misuse. 2011;46(9): 1081-9.

68. Kuramoto SJ, Bohnert AS, Latkin CA. Understanding subtypes of inner-city drug users with a latent class approach. Drug Alcohol Depend. 2011;118(2-3):237-43.

69. Patra J, Fischer B, Maksimowska S, Rehm J. Profiling polysubstance use typologies in a multi-site cohort of illicit opioid and other drug users in Canada-a latent class analysis. Addict Res Theory. 2009;17(2):168-85.

70. Darke S, Hall W, Ross M, Wodak A. Benzodiazepine use and HIV risk-taking behaviour among injecting drug users. Drug Alcohol Depend. 1992;31(1):31-6. 
71. Horyniak D, Agius PA, Degenhardt L, Reddel S, Higgs P, Aitken $\mathrm{C}$, et al. Patterns of, and factors associated with, illicit pharmaceutical opioid analgesic use in a prospective cohort of people who inject drugs in Melbourne, Australia. Subst Use Misuse. 2015;50(13):1650-9.

72. Booth RE, Lehman WEK, Kwiatkowski CF, Brewster JT, Sinitsyna L, Dvoryak S. Stimulant injectors in Ukraine: the next wave of the epidemic? AIDS Behav. 2008;12(4):652-61.

73. Jones JD, Mogali S, Comer SD. Polydrug abuse: a review of opioid and benzodiazepine combination use. Drug Alcohol Depend. 2012;125(1-2):8-18.

74. Sabin KM, Johnston LG. Epidemiological challenges to the assessment of HIV burdens among key populations: respondentdriven sampling, time-location sampling and demographic and health surveys. Curr Opin HIV AIDS. 2014;9(2):101-6.

75. Brecht M-L, Huang D, Evans E, Hser Y-I. Polydrug use and implications for longitudinal research: ten-year trajectories for heroin, cocaine, and methamphetamine users. Drug Alcohol Depend. 2008;96(3):193-201.

76. Degenhardt L, Hall W. Extent of illicit drug use and dependence, and their contribution to the global burden of disease. The Lancet. 2012;379(9810):55-70.
77. Hoffman IF, Latkin CA, Kukhareva PV, Malov SV, Batluk JV, Shaboltas AV, et al. A peer-educator network HIV prevention intervention among injection drug users: results of a randomized controlled trial in St. Petersburg, Russia. AIDS Behav. 2013;17(7):2510-20.

78. Niccolai LM, Verevochkin SV, Toussova OV, White E, Barbour R, Kozlov AP, et al. Estimates of HIV incidence among drug users in St. Petersburg, Russia: continued growth of a rapidly expanding epidemic. Eur J Public Health. 2011;21(5):613-9.

79. Colfax G, Santos G-M, Chu P, Vittinghoff E, Pluddemann A, Kumar S, et al. Amphetamine-group substances and HIV. The Lancet. 2010;376(9739):458-74.

80. Colfax G, Shoptaw S. The methamphetamine epidemic: implications for HIV prevention and treatment. Current HIV/AIDS Rep. 2005;2(4):194-9.

81. MacArthur GJ, Minozzi S, Martin N, Vickerman P, Deren S, Bruneau J, et al. Opiate substitution treatment and HIV transmission in people who inject drugs: systematic review and metaanalysis. BMJ (Online). 2012;345(7879).

82. Malta M, Magnanini MM, Strathdee SA, Bastos FI. Adherence to antiretroviral therapy among HIV-infected drug users: a metaanalysis. AIDS Behav. 2010;14(4):731-47. 